\title{
Recent Trends in Biomarker Research and Development
}

\section{Timothy J. Hagen*}

Department of Chemistry and Biochemistry, Northern Illinois University, DeKalb, Illinois, USA

Biomarkers have tremendous potential to affect the success rate of clinical trials, identify new drug targets and develop diagnostic assays for early detection of disease. The potential positive affect that biomarkers posses, has resulted in a vast number of scientific publications over the past twenty years. A search of the literature from 1990 to 2011 resulted in over 157,000 published scientific publications containing the keyword biomarker [1]. The number or articles have increased dramatically over the past two decades; in 1990, the number of published articles was 182 while in 2011 the number of publications was over 24,000. The areas of biomarker research are far ranging and include drug discovery, toxicity, clinical markers of efficacy and therapeutic index, diagnostic assay development and exposure to environmental pollutants.

Analysis of the root causes of failure of clinical drug candidates is an area of great concern and analysis. In 1991, poor pharmacokinetic (PK) properties were the most important cause of attrition, accounting for about $40 \%$ of all failures [2]. A focused effort on increasing the drugability of clinical candidates had an impact on reduced failure due to PK. By 2000, the attrition rate due to poor PK was down to $10 \%$ [3]. This significant improvement was due to a change in drug discovery strategies, as pharmaceutical companies began assessing PK properties of new chemical entities at the very early stages of drug discovery [4]. Assessment of clinical trial failures in phase II between 2008 and 2010 found that $51 \%$ of the failures were due to a lack of efficacy [5]. Having validated biomarkers that correlate to efficacy will improve success rates in clinical trials. In order for increased success in Phase II clinical trials to become reality, preclinical research that correlates and identifies measureable biomarkers with disease are essential.

Biomarker development originates in the drug discovery phase and leads to clinical validation stage. In the target, discovery and validation stage biomarkers are used to identify and justify targets for therapy [6]. In the lead discovery and optimization phase, biomarkers used to determine target effects and target-associated assays that are used to identify leads. The biomarkers are then used to evaluate the effects of the molecular target. An example was the discovery that the Human Epidermal Growth Factor Receptor 2 (HER2) proto-oncogene was frequently amplified in breast cancer and associated with a poor prognosis [7]. This correlation provided the rationale for anti-HER2 therapeutic strategies that led to the development of trastuzumab [8]. Biomarkers used in preclinical development and lead molecules are developed against targets and further optimized based on biomarker endpoints in model systems or animal studies. In preclinical studies, the development of appropriate animal models that feature biomarker properties comparable with those seen in patient populations will enhance their utility as predictive models. Biomarkers have an essential role in the validation of new disease models.

Biomarkers are critically important to the success of clinical trials. A clinical end-point is defined as a variable that measures how patients feel, function, or survive [5]. A surrogate end-point is a biomarker that is intended to substitute for a clinical end-point. In this case, a surrogate end-point is expected to predict clinical benefit. For example, coronary artery disease is the clinical endpoint for dyslipidemia and a measurable clinical biomarker is elevated LDL cholesterol. The ability to have reliable surrogate endpoint biomarkers will significantly increase success in phase II clinical trials. The pharmaceutical industry has realized this and has incorporated biomarkers into their preclinical and clinical studies. The data management for the vast number of validated biomarkers is improved by the availability of several commercial databases. Thomson Reuter's biomarker database contains over 4000 biomarkers that have approximately 60,000 uses [9]. The FDA realizes the importance of biomarkers and they are seeking to establish a list of proven biomarkers [10]. The FDA categorizes biomarkers into four types. 1) Prognostic biomarkers that are used to determine the progression of a disease in a patient. 2) Predictive biomarkers are baseline biomarkers that indicate the likelihood that a patient will respond to a particular treatment. 3) Pharmacodynamic biomarkers indicate a biological response after receiving a particular drug. An example would be a change in blood pressure after administration of an antihypertensive drug. These biomarkers are generally tested in Phase II clinical trials and submitted to the FDA. 4) Surrogate biomarkers that can be used as a substitute for a clinical efficacy standpoint. This last type of biomarker is controversial because a great deal of evidence may be required. Successful use of biomarkers will have a large impact on increasing the success rate in clinical trials.

Assessment of clinical trial failures in phase II between 2008 and 2010 found that $19 \%$ of the failures were due to safety [5]. Preclinical biomarkers are used to assess toxicity and safety of the drug. The ratio of drug concentrations for efficacy versus toxicity is used to determine a therapeutic index in preclinical models. The translation of animal data to human is the most challenging aspect of these biomarker evaluations. This is an easier task when the toxicity is known and can be easily measured. An example would be a drug that causes an increase in blood pressure as a side effect. The blood pressure can be easily monitored and a therapeutic index determined. The translation between blood pressure in animal and human is often well validated. The development of additional toxicity biomarkers for other major target organs has also been described [11]. Determining toxicity biomarkers is challenging if the mechanism of an observed toxicity is not known [12]. In order for a reliable biomarker for toxicity, research may often be required to determine the mechanism of toxicity and translatability between animal models and humans. Differences in metabolism between humans and animal species used for toxicity evaluations complicate analysis of the data. There is a considerable amount of risk in determination of biomarkers of toxicity.

Another key area that is rapidly increasing is in the area of

*Corresponding author: Timothy J. Hagen , Assistant Professor, Department of Chemistry and Biochemistry, Northern Illinois University, DeKalb, Illinois, USA E-mail: thagen@niu.edu

Received May 21, 2012; Accepted May 23, 2012; Published May 25, 2012

Citation: Hagen TJ (2012) Recent Trends in Biomarker Research and Development. Biochem Anal Biochem 1:e108. doi:10.4172/2161-1009.1000e108

Copyright: (c) 2012 Hagen TJ. This is an open-access article distributed under the terms of the Creative Commons Attribution License, which permits unrestricted use, distribution, and reproduction in any medium, provided the original author and source are credited. 
personalized medicine. The use of genomic data makes it possible to identify subsets of patients that should respond to certain therapies. As biomarker research progresses toward establishing the fundamentals of personalized medicine, the future of drug and biomarker codevelopment resides in identifying the population that would benefit from that drug treatment. The significant effort and resources invested in the development of these biomarkers will expand their roles as surrogate endpoints and diagnostic indicators for disease screening. Successful implementation of biomarkers for personalized medicine will identify a sub-population that should respond well to a certain treatment and reduce risks associated with the drug treatment. This approach will increase the cost and reduce the number of patients prescribed, however these costs should be offset by the increased safety.

Biomarkers can also include a substance whose detection indicates a particular disease state, for example, the detection of an antibody that is a sign of infection. The diagnostics market is well established and has significant unmet medical needs; however, it is highly competitive and has significant entry barriers. In 2011, the worldwide in vitro diagnostic (IVD) market was approximately $\$ 44$ billion that is double what it was in 2001 [13]. The largest segment is immunodiagnostics. Opportunities exist in the early detection of cancer, which could dramatically improve the lives of hundreds of thousands of patients. When bronchial carcinoma is detected, the tumor stage is often advanced and effective therapy is not possible. The overall, five-year survival for all bronchial carcinomas is as low as $5 \%$. If an early diagnosis can be made a survival of, up to $60 \%$ may be possible [14].

Despite these opportunities, the diagnostics market is a challenging. To enter the market, diagnostics need to approval by regulatory agencies that require expensive clinical trials. In order to be successful, diagnostics need to provide demonstrable clinical decision points to physicians. The risk for Alzheimer's disease can be assessed by examining the alleles for apoE, but there are no preventive treatments available so a diagnostic assay is not very useful. The competition is strong with the top 10 diagnostics manufacturers controlling $80 \%$ of the IVD market in the US [15]. These companies are not sitting back and waiting the emergence of new technologies instead, they are actively researching and acquiring companies with innovative products. The challenges and risks of the diagnostics market may not be proportionate to the return. Typical diagnostic assays only generate sales of tens of millions of dollars, whereas therapeutics can generate hundreds of millions or more. [15].

Biomarkers can also be used to indicate exposure to various environmental substances in epidemiology and toxicology. In these cases, the biomarker may be the substance itself, for example asbestos

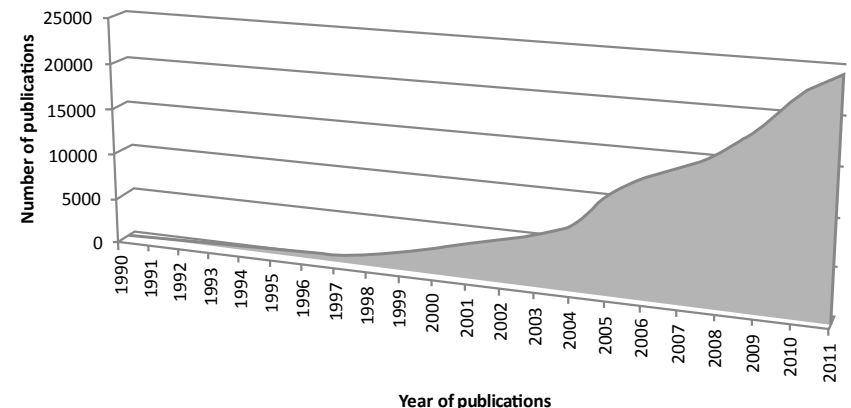

Figure 1: Number of biomarker publications per year. particles or NNK from tobacco, or a variant of the external substance processed by the body such as a metabolite [16]. The use of biomarkers in various species (aquatic animals and invertebrates) may be used to determine the extent and range of environmental pollutants.

It is essential to realize that all biomarkers need to have valid analytical methods. The process begins with choosing the appropriate assay, followed by developing this assay into a validated method. Various types of assays can be used during the biomarker method development and range from the low technology end, for example, Immunohisto chemistry (IHC) to immunoassays to the high technology end that include platforms for genomics, proteomics, and complex ligand binding assays [6]. The need for a standardized approach to the biomarker validation process is becoming increasingly important given the recent rise in the number biomarkers in development.

As biomarker research advances towards personalized medicine, the potential of drug and biomarker development relies on identification the population that would respond to the drug. The significant assets invested in the development of these biomarkers will expand their roles as surrogate endpoints and diagnostics for disease screening, monitoring disease progression and treatment efficacy, and in determining patient outcomes or identifying potential side effects such as in toxicity. The emphasis now should be placed on biomarker assay development and method validation to eliminate the failure of biomarkers that occur in the clinic due to poor assay selection and the lack of validation. Future advances in biomarker research will be focused on the transition of biomarkers from the development phase to clinical applications for drug trials. The revenue that they can generate must balance the cost of developing these biomarkers. The management of the data generated will be a critical factor for success and a central source that is publically available is essential.

\section{References}

1. Scifinder search

2. Roberts SA (2003) Drug metabolism and pharmacokinetics in drug discovery. Curr Opin Drug Discov Devel 6: 66-80.

3. Kola I, Landis J (2004) Can the pharmaceutical industry reduce attrition rates? Nat Rev Drug Discov 3: 711-715.

4. Kerns EH, Di L (2003) Pharmaceutical profiling in drug discovery. Drug Discov Today 8: 316-323.

5. Arrowsmith J (2011) Trial watch: Phase II failures: 2008-2010. Nat Rev Drug Discov 10: 328-329.

6. Chau CH, Rixe O, McLeod H, Figg WD (2008) Validation of analytic methods for biomarkers used in drug development. Clin Cancer Res 14: 5967-5976.

7. Baselga J, Tripathy D, Mendelsohn J, Baughman S, Benz CC, et al. (1996) Phase II study of weekly intravenous recombinant humanized anti-p185HER2 monoclonal antibody in patients with HER2/neu-overexpressing metastatic breast cancer. J Clin Oncol 14: 737-744.

8. Vogel CL, Cobleigh MA, Tripathy D, Gutheil JC, Harris LN, et al. (2002) Efficacy and safety of trastuzumab as a single agent in first-line treatment of HER2overexpressing metastatic breast cancer. J Clin Oncol 20:719-726.

9. Thomson Reuters Integrity: Biomarkers Module.

10. Walker EP (2010) FDA Seeks to Establish List of Proven Biomarkers.

11. Marrer E, Dieterle F (2010) Impact of biomarker development on drug safety assessment. Toxicol Appl Pharmacol 243: 167-179.

12. Bailey WJ, Ulrich R (2004) Molecular profiling approaches for identifying novel biomarkers. Expert Opin Drug Saf 3: 137-151. 
13. In Vitro Diagnostic (IVD) Market (Applications, End-users \& Types) Trends \& Global Forecasts (Major \& Emerging Markets - G7, Japan \& BRIC) (2011 2016).

14. Buszewski B, Schubert J (2011) Biomarkers. Anal Bioanal Chem 401:20372038.
15. Brodovsky J (2000) DCL00 US Clinical Laboratory Diagnostic Market Review, Business Communications, Co.

16. Raza SK, Rao MK (2007) Analysis of Biomarkers by Mass Spectrometry. Proceedings of 12th ISMAS Symposium cum Workshop on Mass Spectrometry Goa, India. 